\title{
Planar crossing numbers of graphs embeddable in another surface
}

\author{
Károly J. Böröczky, János Pach ${ }^{\dagger}$ and Géza Tóth \\ Rényi Institute, Hungarian Academy of Sciences
}

\begin{abstract}
Let $G$ be a graph of $n$ vertices with maximum degree $d$ that can be drawn without crossing in a closed surface of Euler characteristic $\chi$. It is proved that then $G$ can be drawn in the plane with at most $c_{\chi} d n$ crossings, where $c_{\chi}$ is a constant depending only on $\chi$. This result, which is tight up to a constant factor, is strengthened and generalized to the case when there is no restriction on the degrees of the vertices.
\end{abstract}

\section{Introduction and preliminaries}

Let $G$ be a graph with no loops or parallel edges. A drawing or embedding of $G$ on a closed surface $S$ is a representation of $G$ such that the vertices are represented by points and the edges are represented by (possibly crossing) non-selfintersecting continuous arcs in $S$, connecting the corresponding point pairs and not passing through any other vertex. The crossing number (or planar crossing number) of $G, \operatorname{cr}(G)$, is defined as the minimum number of edge crossings over all drawings of $G$ in the plane (or equivalently, on the sphere).

Suppose that $G$ has $n$ vertices and $e$ edges, and suppose that it permits a crossing-free drawing on a closed surface $S$. How large can $\operatorname{cr}(G)$ be? Obviously, we have $\operatorname{cr}(G)<\left(\begin{array}{l}e \\ 2\end{array}\right)$, and this order of magnitude can be attained if $S$ is not the sphere (plane). To see this, take five vertices and connect any pair of them by $\frac{e}{20}$ vertex-disjoint paths of lengths two. Clearly, this graph can be embedded in any closed surface other than the sphere, but no matter how we draw it in the plane, every subdivision of $K_{5}$ gives rise to a crossing. Therefore, the number of crossings must be at least $\frac{e^{2}}{400}$.

*Supported by OTKA-T 049301, 042769, 043520, and by the Marie Curie TOK projects Budalggeo and DiscConvGeo

${ }^{\dagger}$ Supported by NSF grant CCF-05-14079 and grants from NSA, PSC-CUNY, Hungarian Research Foundation, and BSF

${ }^{\ddagger}$ Supported by OTKA-T-038397 and OTKA-T-046246. 
It was conjectured by Peter Brass that this estimate can be substantially improved if we impose an upper bound on the degree of the vertices. This conjecture has been verified in [PT05], for any orientable surface $S$. The aim of this note is to prove Brass' conjecture in full generality.

Theorem 1. Let $G$ be a graph of $n$ vertices with maximum degree $d$, and suppose that $G$ has a crossing-free drawing on the closed surface $S$ of Euler characteristic $\chi$. Then the planar crossing number of $G$ satisfies

$$
\operatorname{cr}(G) \leq 10^{20-7 \chi} d n \text {. }
$$

It is not hard to see that for any fixed $\chi$, this bound is best possible up to a constant factor. For instance, if $S$ is the simplest nonorientable surface, the projective plane, consider the grid graph $H_{k}$ defined on the vertex set $V\left(H_{k}\right)=\{(i, j): 1 \leq i, j \leq k\}$, in which $(i, j)$ and $\left(i^{\prime}, j^{\prime}\right)$ are connected by an edge if and only if $\left|i-i^{\prime}\right|+\left|j-j^{\prime}\right|=1$. Let $H_{k}^{\prime}$ denote the graph obtained from $H_{k}$ by connecting each vertex $(1, j)$ to $(k, k-j+1)$, and each vertex $(i, 1)$ to $(k-i+1, k)$, for all $1 \leq j \leq k$ and $1 \leq i \leq k$. Furthermore, let $G$ be the graph obtained from $H_{k}^{\prime}$ by replacing every edge with $\lceil d / 4\rceil$ paths of length two connecting the same pair of vertices. Clearly, $G$ is a graph of $n=k^{2}(2\lceil d / 4\rceil+1)$ vertices of maximum degree at most $d$, and it can be embedded in the projective plane without any crossing. It is not hard to verify, and follows from a recent result of Gitler, Leanos, and Salazar [GLS06] that $\operatorname{cr}(G) \geq c d n$ holds, for a suitable constant $c>0$. Instead of Theorem 1, we prove a stronger result.

Theorem 2. Let $G$ be a graph of $n$ vertices with degrees $d_{1}, d_{2}, \ldots, d_{n}$, and suppose that $G$ has a crossing-free drawing on a closed surface $S$ of Euler characteristic $\chi$. Then we have

$$
\operatorname{cr}(G) \leq 10^{19-7 \chi} \sum_{i=1}^{n} d_{i}^{2} .
$$

In Section 2, first we reduce Theorem 1 to Theorem 2. Then we reduce Theorem 2 to another statement (Theorem 3 stated there), which, in turn, will be established in the last two sections.

For the proof, we need the well known classification of closed surfaces (that is, compact surfaces with no boundary), discovered in the 1860's [H02], [MT01]. According to this, every closed surface is homeomorphic to one of the surfaces $S_{i}$ or $N_{i}(i=0,1,2, \ldots)$. Here $S_{0}=N_{0}$ is the sphere (plane), and for every $i>0, S_{i}$ and $N_{i}$ can be obtained from $S_{i-1}$ and $N_{i-1}$ by attaching a handle and a crosscap, respectively. The surface $S_{i}$ is orientable, and its Euler characteristic is $\chi\left(S_{i}\right)=2-2 i(i \geq 0)$. The surface $N_{i}$ is nonorientable, and we have $\chi\left(N_{i}\right)=2-i(i>0)$. Hence, the Euler characteristic of any surface is an integer that does not exceed two.

Fix a closed simple curve $\gamma$ in $S$, and consider a small neighborhood of $\gamma$. It is topologically equivalent either to a strip or to a Möbius strip. In the first case, we say that $\gamma$ is orientation preserving, in the second case, it is orientation reversing. The surface $S$ is orientable if and only 
if it contains no orientation reversing curve. If $S \backslash \gamma$ is connected, then $\gamma$ is called a nonseparating curve. Otherwise, $S \backslash \gamma$ consists of two connected components and $\gamma$ is said to be separating.

For the purpose of this paper, we classify the closed curves as follows.

Type 1: $\gamma$ is separating.

In this case, $\gamma$ is necessarily orientation preserving, and any closed curve intersects it an even number of times. Cutting $S$ along $\gamma$, we obtain two surfaces, both bounded by a closed curve. Attach a disk to each of these boundaries. If $\gamma$ was contractible to a point, then we obtain a sphere and a surface homeomorphic to $S$. Otherwise, we obtain two closed surfaces whose Euler characteristics are larger than $\chi(S)$.

Type 2: $\gamma$ is nonseparating and orientation preserving.

In this case, there exists another closed curve which intersects $\gamma$ exactly once. Cutting $S$ along $\gamma$, and attaching a disk to each of the resulting boundary curves, we obtain a compact surface $S^{\prime}$ with Euler characteristic $\chi(S)+2$.

Type 3: $\gamma$ is orientation reversing, and hence nonseparating.

This case can occur only for nonorientable surfaces. There exists another closed curve which intersects $\gamma$ exactly once. Cutting $S$ along $\gamma$, we obtain a surface with one closed boundary curve. Attach a disk to it. The Euler characteristic of the resulting surface $S^{\prime}$ is $\chi(S)+1$. Note that $S^{\prime}$ may be orientable or nonorientable.

For any graph $G$, let $n(G), e(G)$, and $\sigma(G)$ denote the number of vertices of $G$, edges of $G$, and the sum of the squares of their degrees, respectively.

A crossing-free drawing of $G$ on a closed surface $S$ is called a cellular embedding (or a twocell embedding) if every connected component of $S \backslash G$ is homeomorphic to an open disk. It follows from the Euler-Poincare formula that, if $G$ has a cellular embedding in $S$, then we have $e(G) \leq 3 n(G)-3 \chi(S)$.

\section{Reducing Theorems 1 and 2 to Theorem 3}

For both Theorems 1 and 2 we can assume that $G$ is connected, otherwise we can argue separately for its components. We can also assume that $\chi<2$, otherwise there is nothing to prove. Therefore, we have $10^{20-7 \chi} d n>1000 n$ and similarly $10^{19-7 \chi} \sum_{i=1}^{n} d_{i}^{2}>1000 n$. On the other hand, clearly, $\operatorname{cr}(G)<1000 n$ holds for every graph $G$ of at most 10 vertices. Hence we can assume that $n>10$. Suppose that $|\chi| \geq n / 10$. Then we have $10^{20-7 \chi} d n>10^{7 n / 10}>n^{4}>\operatorname{cr}(G)$, and similarly $10^{19-7 \chi} \sum_{i=1}^{n} d_{i}^{2}>10^{7 n / 10}>n^{4}>\operatorname{cr}(G)$, so there is nothing to prove. So, we can and will assume in the sequel that $|\chi| \leq n / 10$. 
First we show that Theorem 2 implies Theorem 1. Consider a crossing-free drawing of $G$ on a closed surface $S$ of maximum Euler characteristic $\chi$. Notice that this must be a cellular embedding. Indeed, if a connected component of $S \backslash G$ is not homeomorphic to a disk, then replacing such a cell by a disk, we obtain a surface $S^{\prime}$ whose Euler characteristic is larger than $\chi$.

Applying the consequence of the Euler-Poincare formula mentioned at the end of Section 1, we obtain that $e(G) \leq 3 n-3 \chi<4 n$.

Let $d_{1} \geq d_{2} \geq \cdots \geq d_{n}$ be the degrees of the vertices. By Theorem 2 , we have

$$
\operatorname{cr}(G) \leq 10^{19-7 \chi} \sum_{i=1}^{n} d_{i}^{2} \leq 10^{19-7 \chi} d_{1} \sum_{i=1}^{n} d_{i} \leq 10^{19-7 \chi} d_{1} \cdot 2 e(G) \leq 10^{19-7 \chi} d_{1} \cdot 10 n=10^{20-7 \chi} d_{1} n,
$$

and Theorem 1 follows $\square$.

Next we turn to the proof of Theorem 2. Recall that $\sigma(G)$ denotes the sum of degrees of the vertices of $G$. In this case, it is sufficient to prove the statement for two-connected graphs. Indeed, if $G$ is disconnected or has a cut vertex, then it can be obtained as the union of two graphs $G_{1}$ and $G_{2}$ with $n_{1}$ and $n_{2}$ vertices that have at most one vertex in common. Arguing for $G_{1}$ and $G_{2}$ separately, we obtain by induction that

$$
\operatorname{cr}(G)=\operatorname{cr}\left(G_{1}\right)+\operatorname{cr}\left(G_{2}\right) \leq 10^{19-7 \chi}\left(\sigma\left(G_{1}\right)+\sigma\left(G_{2}\right)\right) \leq 10^{19-7 \chi} \sigma(G),
$$

as required.

Consider a crossing-free drawing of $G$ on a closed surface $S$ of maximum Euler characteristic $\chi$. As in the proof of Theorem 1, it follows from the maximality that this is a cellular embedding.

If our drawing is not a triangulation, then by adding $O(n)$ extra vertices and edges, we can turn it into one so that the maximum degree of the vertices increases by at most a factor of three. We have to apply the following observation that can be established by a slight modification of the proof of Lemma 2.1 in [PT05], where the same statement is proved under the assumption that $S$ is orientable.

Lemma 2.1. Let $G$ be a two-connected graph with $n$ vertices, each of degree at most $d(d \geq 3)$. Suppose that $G$ has a cellular embedding into a closed surface with Euler characteristic $\chi$. Any such embedding can be extended to a triangulation of $S$ with at most $19 n-18 \chi$ vertices of maximum degree at most $3 d$.

For any graph $G$ and for any $\chi \leq 2$, let $\operatorname{cr}_{\chi}(G)$ be the minimum number of edge crossings over all drawings of $G$ on a surface of Euler characteristic $\chi$. Using this notation, we have that $\mathrm{cr}_{2}(G)=\operatorname{cr}(G)$.

Theorem 2 provides an upper bound on the crossing number of graphs that can be embedded into a surface of Euler characteristic $\chi$. We show that this bound can be deduced by repeated application of the following result. In each step, we increase the Euler characteristic of the surface by at least one. 
Theorem 3. Let $G$ be a two-connected graph with $\mathrm{cr}_{\chi}(G)=0$ for some $\chi<2$. Then we have $\mathrm{cr}_{\chi+1}(G) \leq 10^{5} \sigma(G)$.

Proof of Theorem 2 using Theorem 3. The proof is by reverse induction on $\chi(S)$. Theorem 2 is trivial for $\chi(S)=2$, that is, if $S$ is the sphere. Let $\chi<2$, and suppose that we have already proved Theorem 2 for all $\chi^{\prime}>\chi$. Consider a crossing-free drawing of $G$ on a closed surface $S$ with $\chi(S)=\chi$. According to Theorem $3, G$ can be drawn with at most $10^{5} \sigma(G)$ crossings on another closed surface $S^{\prime}$ of Euler characteristic $\chi\left(S^{\prime}\right) \geq \chi+1$. Place a new vertex at each crossing and denote the resulting graph by $G^{\prime}$. Since every new vertex has degree four, we have

$$
\sigma\left(G^{\prime}\right) \leq \sigma(G)+4^{2} \mathrm{cr}_{\chi+1}(G) \leq \sigma(G)+4^{2} \cdot 10^{5} \sigma(G)=1600001 \sigma(G) .
$$

Applying the induction hypothesis to $G^{\prime}$, we obtain that there exists a drawing of $G^{\prime}$ in the plane with

$$
\operatorname{cr}\left(G^{\prime}\right) \leq 10^{19-7 \chi-7} \sigma\left(G^{\prime}\right) \leq 10^{12-7 \chi} \cdot 1600001 \sigma(G)
$$

crossings. Consider such a drawing, and replace all new vertices of $G^{\prime}$ by a crossing between the corresponding edges of $G$. Then the number of crossings in the resulting planar drawing of $G$ is at most

$$
10^{12-7 \chi} \cdot 1600001 \sigma(G)+10^{5} \sigma(G)<10^{19-7 \chi} \sigma(G),
$$

as required.

It remains to prove Theorem 3 . This will be done in the last two sections.

\section{Proof of Theorem 3: Cutting along a cycle of type 2}

As in Section 1, after Theorem 1, define a grid of size $k \times k$ as the cross product $P_{k} \times P_{k}$ of two paths of length $k$. The boundary of the grid consists of all vertices of $P_{k} \times P_{k}$ whose degree is less than four.

Since $\operatorname{cr}_{\chi^{\prime}}(G) \leq \operatorname{cr}_{\chi}(G)$ for every $\chi^{\prime}>\chi$, to establish Theorem 3, it is sufficient to consider a crossing-free drawing of $G$ in a surface $S$ of maximum Euler characteristic $\chi$. As we have seen at the beginning of Section 2, this implies that we have a cellular embedding of $G$, that is, every cell of $S \backslash G$ is homeomorphic to an open disk. Moreover, since every graph $G$ has a crossing-free drawing on $S_{g}$ with $g=e(G)-1$ [MT01], we can assume that $\chi>-2 e(G) \geq-\sigma(G) / 2$.

In the above cellular embedding of $G$ in $S$, replace each vertex $v$ with degree $d(v)>4$ by a grid of size $d(v) \times d(v)$, lying in a small neighborhood of $v$, and connect the edges incident to $v$ to distinct vertices on the boundary of the grid, without changing their cyclic order. Denote the resulting graph and its embedding in $S$ by $G^{\prime}$. Clearly, $G^{\prime}$ has at most $\sigma(G)$ vertices, each of degree at most four. This is still a cellular embedding, so that we can apply Lemma 2.1 to turn $G^{\prime}$ into a triangulation $G^{\prime \prime}$ with at most

$$
19 \sigma(G)-18 \chi<28 \sigma(G)
$$


vertices, each of degree at most twelve.

Restricting $G^{\prime}$ and $G^{\prime \prime}$ to any grid substituting for a vertex in $G$, the only difference between them is that each quadrilateral face in $G^{\prime}$ is subdivided by one of its diagonals into two triangles in $G^{\prime \prime}$. Color all edges along the boundaries of the grids blue, and all other grid and diagonal edges of $G^{\prime \prime}$ that lie in the interior of some grid red. Color all other edges green. Surface $S$ is not the sphere, so it contains at least one closed curve of type 2 or 3 , that is, at least one nonseparating closed curve (see the end of Section 1). Since $G^{\prime \prime}$ determines a triangulation of $S$, any closed curve is homotopic to a cycle of $G^{\prime \prime}$ (see [MT01]). In particular, $G^{\prime \prime}$ has a nonseparating cycle.

In the rest of this paper, let $C$ denote a fixed cycle of $G^{\prime \prime}$ of type 2 or 3 , with the smallest number $k$ of vertices. In this section, we settle the case when $C$ is of type 2, that is, $C$ is nonseparating and orientation preserving. The other case, when $C$ is of type 3 , will be discussed in Section 4. The two cases of the proof of Theorem 3 are similar. However, they differ at some key points, so in spite of the necessary repetition, they are presented separately.

Lemma 3.1. Let $C$ be of type 2. Then its length $k$ satisfies $k \leq 11 \sqrt{\sigma(G)}$.

Proof of Lemma 3.1. Fix an orientation of $C$ and let $v_{1}, \ldots, v_{k}$ be the vertices of $C$, in cyclic order. Let $E^{l}$ (and $E^{r}$ ) denote the set of edges not belonging to $C$ that are incident to at least one vertex of $C$ and in a small neighborhood of this vertex lie on its left-hand side (respectively righthand side). Replace $C$ by two copies, $C^{r}$ and $C^{l}$, lying on its right-hand side and left-hand side, and denote the vertices of $C^{r}$ and $C^{l}$ by $v_{1}^{r}, \ldots, v_{k}^{r}$ and $v_{1}^{l}, \ldots, v_{k}^{l}$, respectively. Connect each edge of $E^{r}$ and $E^{l}$ to the corresponding vertex of $C_{r}$ and $C_{l}$. Cut $S$ along $C$, and attach two disks to the surface: one along $C_{r}$ and one along $C_{l}$. The resulting surface $S^{\prime}$ satisfies $\chi\left(S^{\prime}\right)=\chi(S)+2$, and it contains a crossing-free drawing $G_{1}$ of a graph slightly different from $G^{\prime \prime}$. Contract $v_{1}^{r}, \ldots, v_{k}^{r}$ resp. $v_{1}^{l}, \ldots, v_{k}^{l}$ into a new vertex $V^{r}$ resp. $V^{l}$. If during this procedure multiple edges are create, remove all repetitions, that is, keep only one edge between any pair of points. Denote the resulting graph by $G_{2}$. It has at most $28 \sigma(G)$ vertices, and it is drawn on $S^{\prime}$ as a triangulation.

Claim 3.2. There is no path of length smaller than $k / 2$ in $G_{2}$, connecting $V^{r}$ and $V^{l}$.

Proof. Suppose for contradiction that there is such a path $P$. It corresponds to a path $P$ of the same length connecting some $v_{i}^{r}$ and $v_{j}^{l}$ in $G_{2}$. Clearly, $i$ and $j$ are at distance at most $k / 2$ in the cyclic order. Assume without loss of generality that $i \leq j \leq i+k / 2$. Extend the path $P$ by adding $v_{j}^{l} v_{j-1}^{l} \ldots v_{i}^{l}$. The new path, denoted by $\tilde{P}$, connects $v_{i}^{r}$ to $v_{i}^{l}$ and it has length $\tilde{l}<k$. Clearly, $\tilde{P}$ corresponds to a cycle in $G^{\prime \prime}$ with the same length $\tilde{l}<k$. Since this cycle intersects $C^{r}$ exactly once, very close to $v_{i}$, it is nonseparating, therefore, it is either of type 2 or of type 3 . This is a contradiction, because, according to our assumption, $G^{\prime \prime}$ has no nonseparating cycle shorter than $C$.

Return to the proof of Lemma 3.1. By Menger's theorem, the maximum number $p$ of (internally) vertex-disjoint paths connecting $V^{r}$ and $V^{l}$ in $G_{2}$ is equal to the minimum number of vertices whose deletion separates $V^{r}$ from $V^{l}$. Choose $p$ such separating vertices $u_{1}, \ldots, u_{p}$, and 
let $K$ denote the set of all edges incident to $u_{1}, \ldots, u_{p}$. With a slight abuse of notation, we use the same letter $K$ to denote the union of these edges, as a point set, including the endpoints of the edges.

Claim 3.3. The set $K$ contains a nonseparating cycle in $S$, whose length is at most $2 p$.

Proof. Suppose that there is a curve $\delta$ connecting $V^{r}$ and $V^{l}$ in $S^{\prime}$, which does not meet $K$. Orient $\delta$ towards $V^{l}$. Let $e_{1}, \ldots, e_{q}$ denote the sequence of edges of $G$, in the order they are intersected by $\delta$, and let $w_{1}, \ldots, w_{q}$ denote the right endpoints of these edges, with respect to the orientation of $\delta$. Using the fact that $\tilde{G}$ is a triangulation, we obtain that for every $i, 1 \leq i<q$, the points $w_{i}$ and $w_{i+1}$ are either identical or adjacent. Therefore, $V^{r} w_{1} w_{2} \ldots w_{q} V^{l}$ is a path in $G_{2}$ connecting $V^{r}$ to $V^{l}$. Using the fact that $\delta$ does not intersect $K$, this path must avoid all edges incident to the separating vertices $u_{1}, \ldots, u_{p}$. Thus, $V^{r} w_{1}, \ldots, w_{q} V^{l}$ avoids the points $u_{1}, \ldots, u_{p}$, a contradiction.

Thus, we can conclude that $K$ contains a cycle that separates $V^{r}$ and $V^{l}$ on the surface $S^{\prime}$. Since every edge that belongs to $K$ is incident to at least one of the points $u_{1}, \ldots, u_{p}$, the length of shortest such cycle $\tilde{C}$ is at most $2 p$. We claim that $\tilde{C}$ is a nonseparating cycle in $S$. By the minimality of $\tilde{C}$, there is a curve $\gamma$ connecting $V^{r}$ and $V^{l}$ on $S^{\prime}$, which intersects $\tilde{C}$ exactly once. As in the proof of Claim 3.2, we can use $\gamma$ and $C$ to construct a closed curve on $S$, which intersects $\tilde{C}$ exactly once. This implies that $\tilde{C}$ is a nonseparating cycle in $S$.

Now we are in a position to complete the proof of Lemma 3.1. Since the length of the shortest nonseparating cycle (in $G^{\prime \prime} \subset S$ ) is $k$, it follows from Claim 3.3 that there are $p \geq k / 2$ vertexdisjoint paths connecting $V^{r}$ and $V^{l}$. By Claim 3.2, the length of each of them is at least $k / 2$. Using the fact that $G^{\prime \prime}$ has at most $28 \sigma(G)$ vertices, we can conclude that $k^{2} / 4 \leq 28 \sigma(G)$, so that $k \leq 11 \sqrt{\sigma(G)}$, as required. The proof of Lemma 3.1 is complete.

Without increasing the length of $C$ too much, we can replace all of its red edges by blue ones. Indeed, the first vertex and the last vertex of any maximal red path in $C$ must belong to the boundary of the same grid. Replace each such path by the shortest blue path connecting its first and last vertices along the boundary of the grid containing them. The resulting blue cycle $C^{\prime}$ is also of type 2 , and its length $m$ is at most $2 k$. We can assume without loss of generality that $C^{\prime}$ does not intersect itself. Fix an orientation of $C^{\prime}$.

Proceed now with $C^{\prime}$ as we did with $C$ in the proof of Lemma 3.1. To simplify the notation, let us again denote by $E_{l}$ (by $E_{r}$ ) the set of edges not belonging to $C^{\prime}$, which are incident to at least one vertex of $C^{\prime}$ and in a small neighborhood of this vertex lie on the left-hand side (right-hand side) of $C^{\prime}$. Replace again $C^{\prime}$ by two of its copies, $C_{r}$ and $C_{l}$, lying on its right-hand side and left-hand side, and denote the vertices of $C^{r}$ and $C^{l}$ by $v_{1}^{r}, \ldots, v_{m}^{r}$ and $v_{1}^{l}, \ldots, v_{m}^{l}$, respectively. Connect every edge of $E_{r}$ (and $E_{l}$ ) to the corresponding vertex of $C_{r}$ (and $C_{l}$ ). Cut $S$ along $C$, and attach to the surface a disk along each side of the cut. For the resulting surface $S^{\prime}$, we have $\chi\left(S^{\prime}\right)=\chi(S)+2$, and $S^{\prime}$ contains a crossing-free drawing $G_{1}$ of a graph slightly different from 
$G^{\prime \prime}$. (Note that $G_{1}$ may be different from the drawing we denoted by $G_{1}$ in the proof of Lemma 3.1.) To obtain a drawing of $G^{\prime \prime}$ from $G_{1}$, we have to remove $C_{l}$ and (re)connect the edges of $E_{l}$ to the corresponding vertices of $C_{r}$, without creating too many crossings.

As before, contract $v_{1}^{r}, \ldots, v_{m}^{r}$ and $v_{1}^{l}, \ldots, v_{m}^{l}$ into two new vertices, $V^{r}$ and $V^{l}$, and remove the possibly arising repeated edges. The resulting graph $G_{2}$ has at most $28 \sigma(G)$ vertices, and it is drawn on $S^{\prime}$ as a triangulation. By Menger's theorem, the maximum number $p$ of vertex-disjoint paths connecting $V^{r}$ and $V^{l}$ in $G_{2}$ is equal to the minimum number of vertices whose deletion separates $V^{r}$ from $V^{l}$, and again we have $p \geq k / 2$. Therefore, the length of the shortest connecting path is at most $28 \sigma(G) / p \leq 56 \sigma(G) / k$. Such a shortest path $P$ corresponds to a path of the same length connecting some vertex $v_{i}^{r}$ to a vertex $v_{j}^{l}$ in $G_{1}$. As before, we can replace all red edges of $P$ by blue edges, without increasing its length by more than a factor of two. Let $P^{\prime}$ denote the resulting blue path, whose length $\ell$ is therefore smaller than $112 \sigma(G) / k$.

Delete the vertices of $C_{l}$. We want to use $P^{\prime}$ as a "corridor" through which we can "pull" all edges in $E_{l}$, and connect them to the corresponding vertices of $C_{r}$. Observe that for every vertex $v$ of $P^{\prime}$, all red edges incident to $v$ are on the same side of $P^{\prime}$. Therefore, when we extend the edges of $E_{l}$ along $P^{\prime}$, we can avoid crossing any red edge. To achieve this, the extended edges of $E_{l}$ may have to cross $P^{\prime}$ several times.

Let us count the number of crossings in the resulting drawing. Each vertex of $C^{\prime}$ has degree at most 12 , so it is adjacent to at most 10 edges of $E_{l}$. Since $\left|C^{\prime}\right| \leq 2 k$, we have $\left|E_{l}\right| \leq 20 k$. Pulling the edges in $E_{l}$ along $P^{\prime}$, we can create at most 12 crossings per edge, in the neighborhood of each vertex of $P^{\prime}$. The total number of these crossings is at most $12 l \cdot 20 k \leq 26880 k \cdot \sigma(G) / k=$ $26880 \sigma(G)$. In addition, we may have to create some crossings between the edges of $E_{l}$. The number of such crossings cannot exceed $\left(\begin{array}{c}\left|E_{l}\right| \\ 2\end{array}\right)$. Using Lemma 3.1, we obtain that $\left(\begin{array}{c}\ell \\ 2\end{array}\right) \leq\left(\begin{array}{c}20 k \\ 2\end{array}\right)<$ $24200 \sigma(G)$. Summarizing, during the whole procedure we created at most $X=51080 \sigma(G)$ crossings.

Deleting the extra vertices and edges from $G_{1}$ and collapsing each grid into a vertex, we obtain a drawing of $G$ on $S^{\prime}$, in which the number of crossings cannot exceed $X$. This concludes the proof of Theorem 3 in the case when the shortest cycle $C \subset G^{\prime \prime}$ homotopic to $\gamma$ was of type 2 .

\section{Proof of Theorem 3: Cutting along a cycle of type 3}

It remains to prove Theorem 3 in the case when the shortest cycle $C \subset G^{\prime \prime}$ homotopic to $\gamma$ is of type 3, that is, orientation reversing, and hence nonseparating. (See the beginning of Section 3, above Lemma 3.1.)

Lemma 4.1. Let $C$ be of type 3. Then its length $k$ satisfies $k \leq 35 \sqrt{\sigma(G)}$.

Proof of Lemma 4.1. Let $v_{1}, \ldots, v_{k}$ denote the vertices of $C$, in cyclic order. Suppose for simplicity that $k$ is divisible by four. Contract $v_{1}, \ldots v_{k / 4-1}$ into a new vertex $V_{1}$, contract 

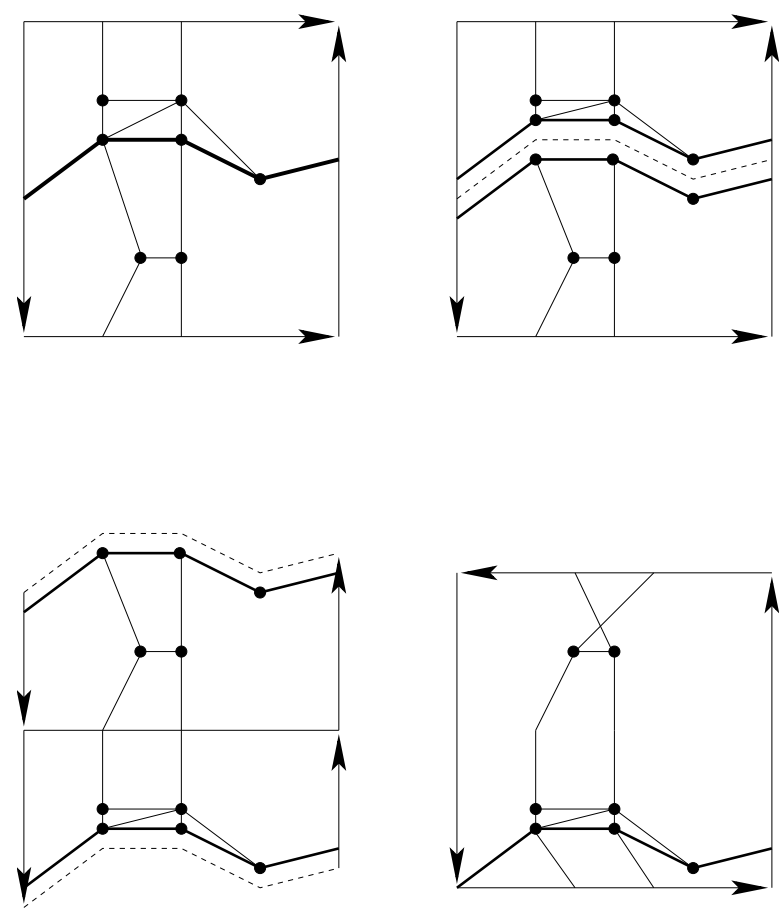

Figure 1: Cut the Klein bottle along a cycle of type 3 to get a drawing in the projective plane.

$v_{k / 4+1}, \ldots v_{k / 2-1}$ into a vertex $V_{2}$, contract $v_{k / 2+1}, \ldots v_{3 k / 4-1}$ into $V_{3}$, and $v_{3 k / 4+1}, \ldots v_{k-1}$ into $V_{4}$. Remove the possibly arising multiplicities by keeping only one edge between any pair of vertices. The resulting graph $\tilde{G}$ has at most $28 \sigma(G)$ vertices, and it is drawn on $S$ as a triangulation. Let $\tilde{C}$ denote the cycle $V_{1} v_{k / 4} V_{2} v_{k / 2} V_{3} v_{3 k / 4} V_{4} v_{k} \subset \tilde{G}$ that corresponds to $C \subset G$. Clearly, $\tilde{C}$ is also orientation reversing.

Claim 4.2. (i) There is no path of length smaller than $k / 4$ in $\tilde{G} \backslash\left\{V_{2}, V_{4}\right\}$, connecting $V_{1}$ and $V_{3}$.

(ii) There is no path of length smaller than $k / 4$ in $\tilde{G} \backslash\left\{V_{1}, V_{3}\right\}$, connecting $V_{2}$ to $V_{4}$.

Proof. (i) Suppose for contradiction that there is such a path $\tilde{P}$. It corresponds to a path $P$ of the same length connecting $v_{i}$ and $v_{j}$ in $G$, for some $1 \leq i \leq k / 4-1, k / 2+1 \leq j \leq 3 k / 4-1$. The vertices $v_{i}$ and $v_{j}$ divide $C$ into two intervals. One of these intervals together with $P$ forms an orientation reversing cycle, which is shorter than $C$, a contradiction.

Part (ii) of the claim can be proved analogously. 
Return to the proof of Lemma 4.1. By Menger's theorem, the maximum number $p$ of vertexdisjoint paths connecting $V_{1}$ and $V_{3}$ in $\tilde{G}$ is equal to the minimum number of vertices whose deletion separates $V_{1}$ from $V_{3}$. Choose $p$ such separating vertices, $u_{1}, \ldots, u_{p}$. As in the proof of Lemma 3.1, let $K$ stand for the collection of all edges incident to $u_{1}, \ldots, u_{p}$, as well as the closed point set obtained by taking the union of these edges.

Claim 4.3. There is a path of length at most $2 p$ in $K$, connecting $V_{2}$ and $V_{4}$.

Proof. Suppose that there is a curve $\delta$ connecting $V_{1}$ and $V_{3}$, which is disjoint from $K$. Orient $\delta$ towards $V_{3}$. Let $e_{1}, \ldots, e_{q}$ denote the sequence of edges of $G$ intersected by $\delta$, and let $w_{1}, \ldots, w_{q}$ denote the right endpoints of these edges, with respect to the orientation of $\delta$. Since $\tilde{G}$ is a triangulation, it follows that for every $i, 1 \leq i<q$, the vertices $w_{i}$ and $w_{i+1}$ are either identical or adjacent. Therefore, $V_{1} w_{1} w_{2} \ldots w_{q} V_{3}$ is a path in $\tilde{G}$ connecting $V_{1}$ and $V_{3}$. By definition, $\delta$ does not intersect $K$, hence the path $V_{1} w_{1}, \ldots, w_{q} V_{3}$ avoids all points $u_{1}, \ldots, u_{p}$, which is impossible. Thus, $K$ separates $V_{1}$ and $V_{3}$ on the surface $S$. The separating set $\left\{u_{1}, \ldots, u_{p}\right\}$ must block $V_{1} v_{k / 4} V_{2} v_{k / 2} V_{3}$ and $V_{1} v_{k} V_{4} v_{3 k / 4}$, the pair of paths connecting $V_{1}$ and $V_{3}$ along $\tilde{C}$. It follows that $K$ contains both $V_{2}$ and $V_{4}$, and hence a whole path connecting $V_{2}$ and $V_{4}$. Every edge that belongs to $K$ incident to at least one $u_{i}(1 \leq i \leq p)$. This yields that the length of any simple path in $K$ between $V_{2}$ and $V_{4}$ is at most $2 p$.

To complete the proof of Lemma 4.1, recall that $p$ is the maximum number of vertex-disjoint paths connecting $V_{1}$ and $V_{3}$ in $\tilde{G}$. One of them passes through $V_{2}$ and one of them through $V_{4}$. By Claim 4.2(i), each of the other $p-2$ paths has length at least $k / 4$. According to Claim 4.3, there is a path connecting $V_{2}$ and $V_{4}$ in $\tilde{G} \backslash\left\{V_{1}, V_{3}\right\}$, whose length is at most $2 p$. By Claim 4.2 (ii), its length is at least $k / 4$, so we have $p \geq k / 8$. Therefore, $28 \sigma(G) \geq(k / 8-2) \cdot k / 4$, so $\sqrt{28 \cdot 32 \sigma(G)} \geq k-16$. Since $G$ has at least 10 vertices and it is two-connected, we have that $\sigma(G) \geq 40$, and then it follows by a routine calculation that $k<35 \sqrt{\sigma(G)}$. This concludes the proof of Lemma 4.1.

The end of the proof is very similar to the end of the proof (described in Sectiom 3) in the case when $C$ is of type 2 . Without increasing the length of $C$ by more than a factor of two, we can replace all red edges of $C$ by blue edges. Denote by $C^{\prime}$ the resulting blue cycle of length at most $2 k$, and fix an orientation of $C^{\prime}$. Let $E_{l}$ (and $E_{r}$ ) denote the set of edges not belonging to $C^{\prime}$ that are incident to at least one vertex of $C^{\prime}$ and in a small neighborhood of this vertex lie on the left-hand side (right-hand side) of $C^{\prime}$. Replace each vertex $v_{i}$ of $C$ by two copies, $v_{i}^{l}$ and $v_{i}^{r}$, on the left-hand and right-hand sides of $C^{\prime}$, respectively. Connect each edge of $E_{r}$ and $E_{l}$ ) to the corresponding vertex $v_{i}^{r}$ or $v_{i}^{l}$.

Cut $S$ along $C$. Since $C$ is an orientation reversing cycle, we obtain one boundary curve. Attach a disk to the surface along this boundary curve. The resulting surface $S^{\prime}$ has Euler characteristic $\chi\left(S^{\prime}\right)=\chi+1$, and it contains a crossing-free drawing $G_{1}$ of a graph slightly different from $G^{\prime \prime}$. To obtain a drawing of $G^{\prime \prime}$ from $G_{1}$, we have to remove $v_{1}^{l}, \ldots, v_{k}^{l}$ and (re)connect the 
edges of $E_{l}$ to the corresponding vertices among $v_{1}^{r}, \ldots, v_{k}^{r}$, without creating too many crossings. Since each vertex has degree at most twelve, we have to reconnect at most $12 k$ edges. Clearly, this can be done within the disk attached to the surface along the cut, creating at most one crossing between any pair of edges and no crossing with the other edges of $G^{\prime \prime}$. Therefore, the total number of crossings is at most

$$
X \leq\left(\begin{array}{c}
12 k \\
2
\end{array}\right)<72 k^{2}<72 \cdot 35^{2} \sigma(G)<90000 \sigma(G)
$$

Deleting the extra vertices and edges from $G^{\prime \prime}$ and collapsing each grid into a vertex, we obtain a drawing of $G$ on $S^{\prime}$, in which the number of crossings cannot exceed $X$. This concludes the proof of Theorem 3 in the case when $C$ is of type 3 .

Remark. The coefficients $10^{20-7 \chi}$ and $10^{19-7 \chi}$ in Theorems 1 and 2 , respectively, are very far from being optimal. With a slightly more careful calculation they can be reduced to significantly smaller exponential functions, but it is very likely that the optimal coefficients are polynomials, or even linear functions of $\chi$.

Acknowledgement. We would like to thank Zoltán Szabó (Princeton), András Stipsicz, and Gábor Tardos (Rényi Institute) for many valuable suggestions. We are also grateful to David Wood (UPC Barcelona), who found a small gap in our original argument outlined in [PT05]. Recently, Wood and Telle [WT06] managed to extend some of our techniques and results to graphs with a fixed excluded minor. They conjecture that Theorem 2 remains true in this more general setting.

\section{References}

[GLS06] I. Gitler, J. Leanos, and G. Salazar: The crossing number of a projective graph is quadratic in the face-width. (Manuscript)

[H02] A. Hatcher: Algebraic topology. Cambridge University Press, Cambridge, 2002. Section 3.2.

[MT01] B. Mohar and C. Thomassen: Graphs on surfaces. Johns Hopkins Studies in the Mathematical Sciences. Johns Hopkins University Press, Baltimore, MD, 2001.

[PT05] J. Pach and G. Tóth: Crossing number of toroidal graphs. In: Graph Drawing, Lecture Notes in Computer Science 3843, Springer-Verlag, Berlin, 2006, 334-342.

[WT06] D. R. Wood and J. A. Telle: Planar decompositions and the crossing number of graphs with an excluded minor, arXiv:math.CO/060446\%. 